\title{
Energy transfer process between two laser compounds coumarin 334
}

\section{\& rhodamine 590}

\author{
Nathera Abass Ali AL Tememee, Noor Zuheir Habeeb \\ Department of Physics, Collage of Science, University of Baghdad \\ E-mail: nathera_2007@yahoo.com
}

\begin{abstract}
In the current research the absorption and fluorescence spectrum of Coumarin (334) and Rhodamine (590) in ethanol solvent at different concentration $\left(10^{-3}, 10^{-4}, 10^{-5}\right) \mathrm{M}$ had been studied. The absorption intensity of these dyes increases as the Concentration increase in addition to that the spectrum was shifted towards the longer wavelength (red shift). The energy transfer process has been investigated after achievement this condition. The fluorescence peak intensity of donor molecule was decrease and its bandwidth will increases on the contrary of the acceptor molecule its intensity increase gradually and its bandwidth decreases as the acceptor concentration increase.
\end{abstract}

Key words

Energy transfer, laser compounds, dye spectrum.

Article info.

Received: May. 2016

Accepted: Jun. 2016

Published: Dec. 2016

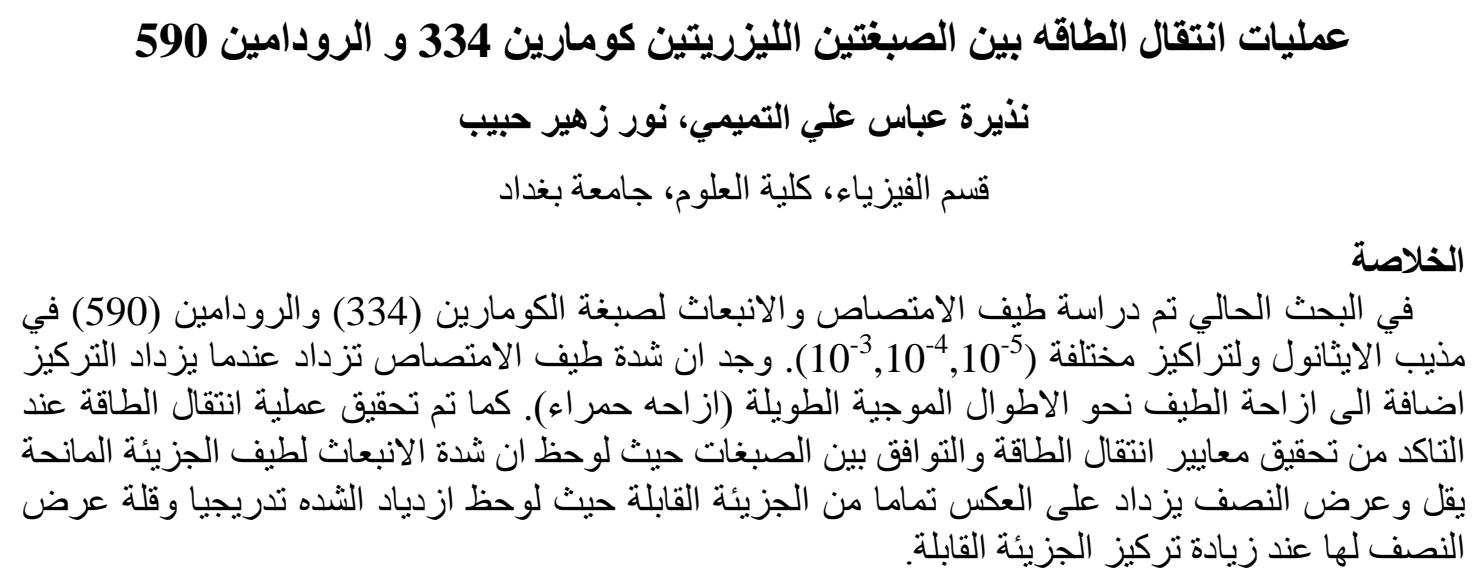

\section{Introduction}

Energy transfer between molecules is a core phenomenon in photosynthesis [13] and an enabling technology for photovoltaic [4, 5], organic lighting sources [6] or bio sensing [7]. Electronic energy transfer in a dye mixture is established as an additional effective mechanism to extend the wavelength of lasing and tunability in an energy transfer dye laser (ETDL). For such dye laser an enhancement or decrease in the laser energy output is noticed .An ETDL with wide tunability has been achieved using two, three, and four component dye mixtures. The most frequently used donor-acceptor pairs in the ETDL are: crestylvioletrhodamine 6G, rhodamine B-DODC, Coumarin - acrivlavine, different Coumarin dyes with some aromatic compounds [8].

Treansfer of the electronic excitation energy from the donor molecule (D) to an acceptor molecule (A) generally occurs in one of the following processes: (a) radiative transfer, (b) Förster energy resonance transfer (FRET), (c) collisional energy transfer, or (d) molecular complexation [12-15]. 
The radiative energy transfer has a great importance when the concentration of the donor and acceptor is low, then the distance between molecules will be large comparing to collision diameter $\left(150<R_{0}\right) A^{0}[10]$, i.e. there is no direct reaction between the donor molecule (D)and the acceptor molecule (A). In other word the radiative transfer of energy is confined to the acceptor molecules which the change of spin multiplicity doesn't happen in, i.e. spin permissible transfer, like energy transfer (mono-mono) or (tri-tri). In relation to the probability of the transfer (mono-tri), it's very weak because the absorption parameter of it is waek [8], while föster resonance energy transfer (FRET) is a mechanism describing energy transfer between a donor molecule (D) in an excited electronic state to an acceptor molecule (A)[6]. The donor molecules typically emit at shorter wavelength which overlap with the absorption spectrum of the acceptor. The energy is not emitted by the donor (D) as a photon, or absorbed as a photon by the acceptor (A), but is transferred by nonradiative pathways [10]. The perturbation of the excited (D) molecule on the (A) molecule take place electrodynamically through space, as a dipole-dipole interaction. This interaction only takes place over a 0.5-10 $\mathrm{nm}$ range, limiting the distance over which (FRET) can occur. The rate of transfer of energy is a strong function of separation distance, and is proportional to (distance) $)^{-6}$. The distance at which the (FRET) efficiency is $50 \%$ is known as the Föster radius $\left(\mathrm{R}_{0}\right)$ [11] and is typically in the range of 2-6 $\mathrm{nm}$. Suitable donor/acceptor pairs require:

-sufficient spectral overlap of the emission and absorption spectra.

-High quantum yield of donor.

-High absorption coefficient of acceptor.

-Matching orientations of the donor and acceptor dipoles.

In order for (FRET) to occur the acceptor does not have to be fluorescent, but if so, it emits a photon following transfer, which is characteristic of the acceptor fluorescence spectrum, although only $\mathrm{D}$ has been initially excited. Fig. 1 shows this process for the ideal case of two molecules with a large stokes shift. (A): illustrates that if the distance between the donor and acceptor molecule is beyond the (FRET) limit and the system is excited, donor emission can be observed but no energy transfer occurs and there is no emission of the acceptor. However when the donor and acceptor are within the (FERT) limit energy transfer can occur and both donor and acceptor emission can now be seen, with the donor emission reduced. (B): Shows how the emission spectra of the donor and acceptor change for (non-FRET) and (FRET). (C): Shows the lifetime of the donor changes for (non -FRET) and (FRET), where a reduction in lifetime occurs. The fluorescence lifetime of a fluorophore depends on both radiative and non-radiative processes. Energy transfer from the donor molecule to the acceptor molecule causes an increase in the non-radiative rate and a decrease in the radiative rate of the donor, this result in a decreased lifetime of the donor. 


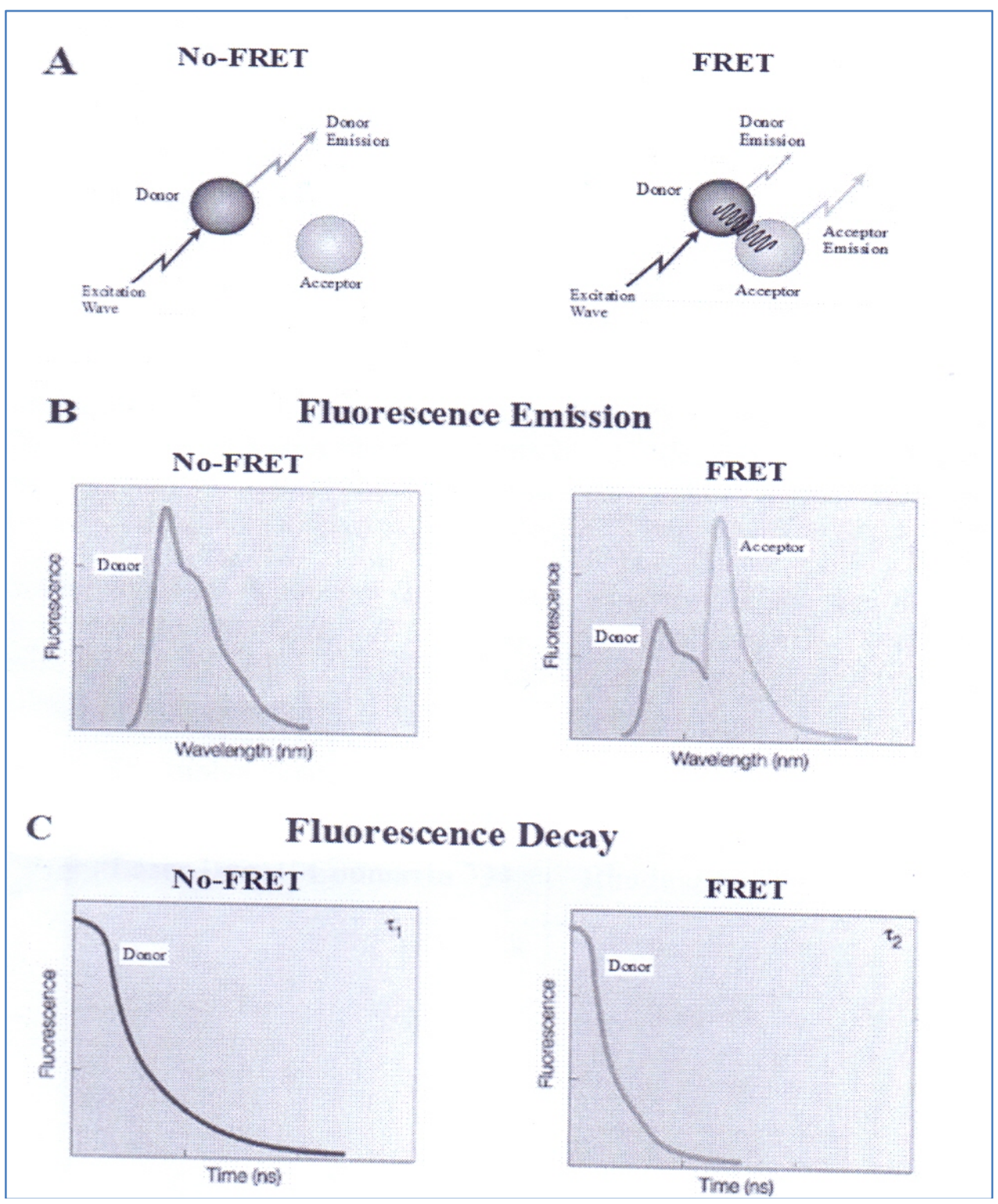

Fig. 1: Fluorescence emission and fluorescence decay spectra of (non-FRET) and (FRET) systems. (A): Donor and acceptor molecule interaction, (B): Fluorescence emission for (non-FRET) and (FRET), (C): Fluorescence decay for (non-FRET) and (FRET). Adapted from [12].

The energy transfer mechanism appearing in a given dye mixture depends on the fluorescence characteristics of the donor and acceptor and, in many cases is influenced by the method of excitation.

\section{Materials and methods}

1- Preparation of fluorescent dyes The structure of the fluorophore examined in this study are shown in Fig. 2 (coumarin 334, Rhodamin 590). 


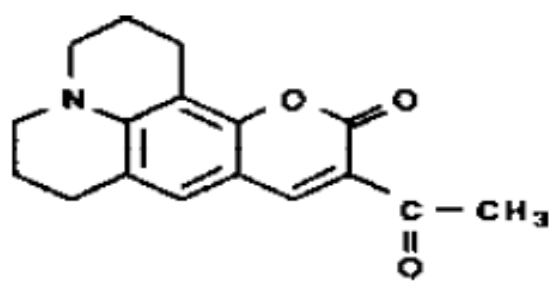

Coumarin 334

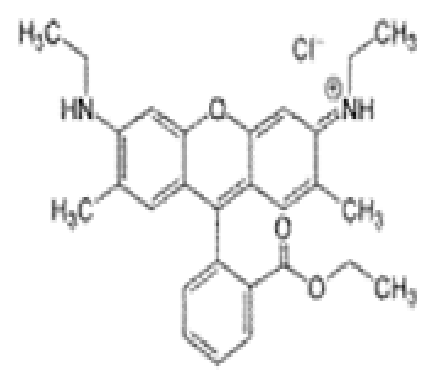

Rhodamin 590

Fig. 2: Sitructures of the examined dyes.

The scientific name of Coumarin 334 is $2,3,5,6-1 \mathrm{H}, 4 \mathrm{H}$-Tetrahydro-9acetylquinolizino-[9, 9a, 1-gh]coumarin - coumarin 521 and its molecular wheight $283.33(\mathrm{gm} / \mathrm{cm} 3)$ with a chemical formula $\mathrm{C}_{17} \mathrm{H}_{17} \mathrm{NO}_{3}$, while Rhodamine 590 is a synthetic organic compound available as a dark reddish purple, brown or black crystalline solid, its molecular formula
$\left(\begin{array}{lllll}\mathrm{C}_{28} & \mathrm{H}_{31} & \mathrm{~N}_{2} & \mathrm{O}_{3} & \mathrm{Cl}\end{array}\right)$, molar mass (479.03 g/mol) Each dye was dissolved in ethanol (polar protic, its dielectric constant $\varepsilon=25$, and viscosity g (mpa s) $\left(20 C^{0}\right)$ is 1.2 to create a stock solution. Dye concentrations were ranged from $0.5^{*} 10^{-4} \mathrm{M}$ to $2 * 10^{-4} \mathrm{M}$ but the molar concentration of the Mixing laser dyes are listed in Table 1.

Table 1: The molar concentration of the mixing prepared dye solutions.

\begin{tabular}{|c|c|c|}
\hline Laser Dye & Coumarin 334 & Rhodamine 590 \\
\hline \multirow{10}{*}{$\begin{array}{c}\text { Concentration } \\
\text { mole/litter }\end{array}$} & \multirow{10}{*}{$\begin{array}{c}5 \times 10^{-4}, 2 \times 10^{-4} \& \\
0.5 \times 10^{-4}\end{array}$} & $0.16 \times 10^{-4}$ \\
\hline & & $0.28 \times 10^{-4}$ \\
\hline & & $0.37 \times 10^{-4}$ \\
\hline & & $0.44 * 10^{-4}$ \\
\hline & & $0.5 \times 10^{-4}$ \\
\hline & & $0.54 \times 10^{-4}$ \\
\hline & & $0.58 \times 10^{-4}$ \\
\hline & & $0.61 \times 10^{-4}$ \\
\hline & & $0.64 \times 10^{-4}$ \\
\hline & & $0.66 \times 10^{-4}$ \\
\hline
\end{tabular}

\section{Experimental setup}

UV-Visible absorption spectra of the laser compounds were carried. The absorption spectra of prepared sample were obtain using UV-VIS mate SP 8001 double beam spectrophotometer covering the range (190-1100nm) supplied by Meterech Corporation (Taipei, Taiwan). Quartiz cell (standard cell), was used for testing liquid samples. the fluorescence spectrum at room temperature in the emission wavelength range (180900)nm was measured using fluorescence spectrophotometer, spectra Academy L td SV-2100 made in Korea .....as shown in Fig. 3. 

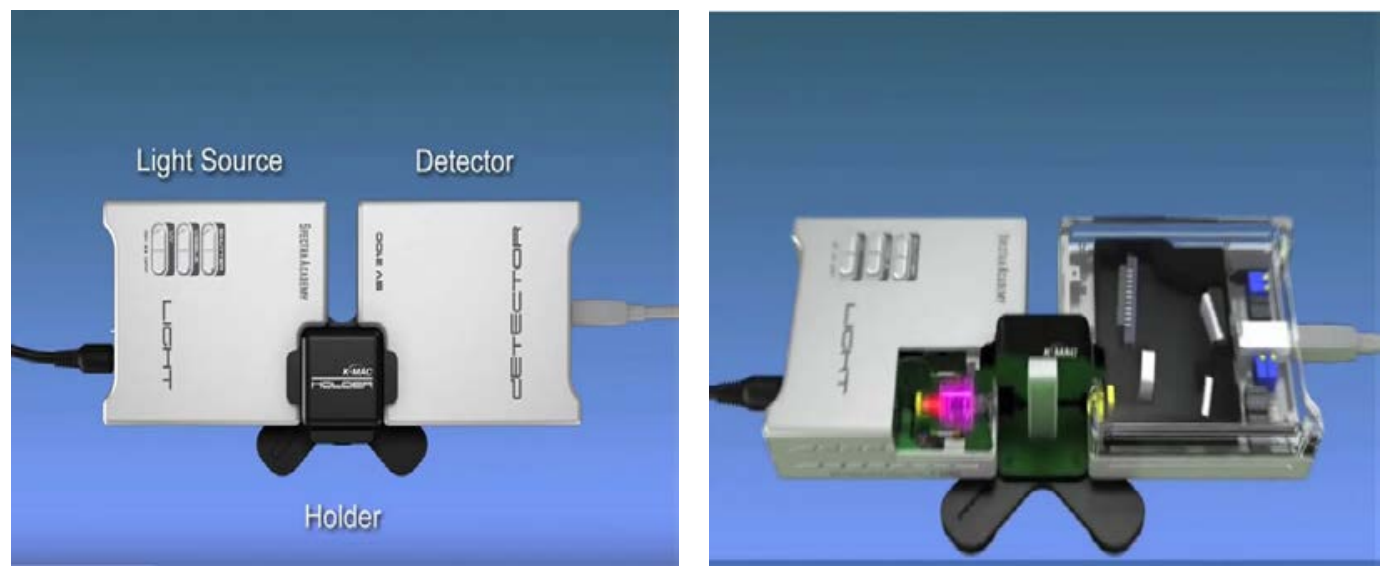

Fig. 3: The fluorescence spectrophotometer.

\section{Result and discussion}

The normalizing absorption spectra of coumarin 334 and Rhodamine 590 dyes solution (at different

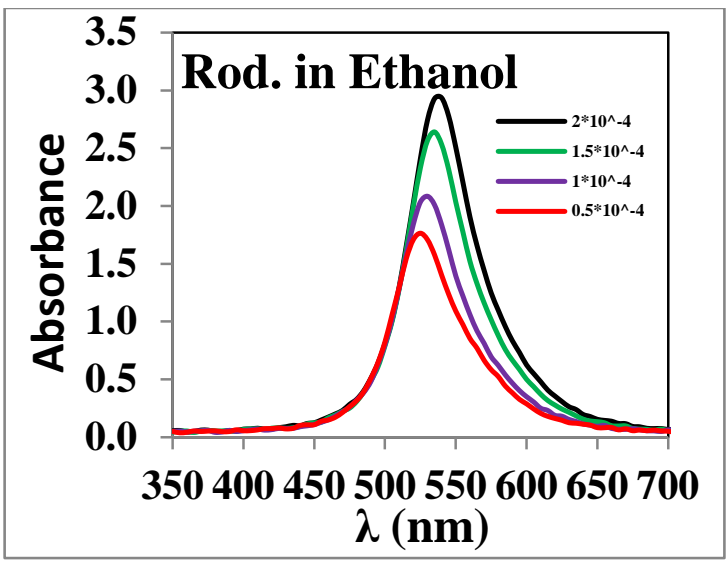

concentration) are shown in Fig. 4. The peak absorption of Coumarin 334 and Rhodamine 590 dye are around (425-463) $\mathrm{nm}$ and (523-536) $\mathrm{nm}$.

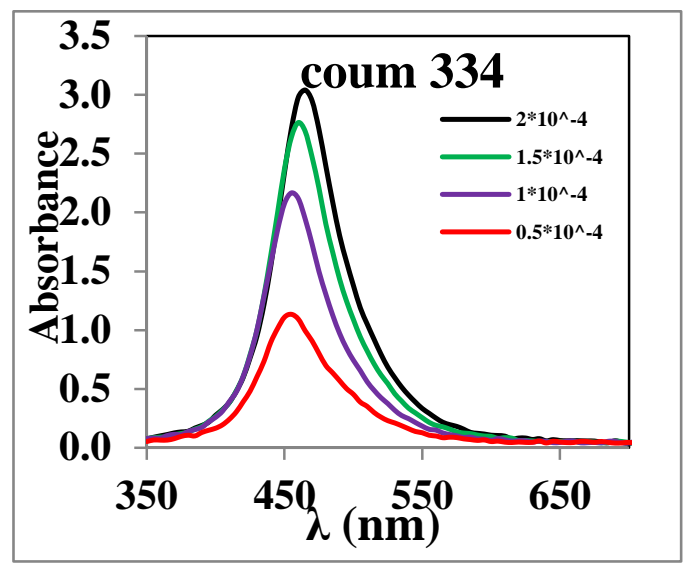

Fig. 4: The absorption spectrum of (a) Coumarin 334 and (b) Rhodamine 590 dye solution.

Fig. 5 shows the normalized fluorescence spectra of Coumarin 334 and Rhodamine 590 the peak fluorescence of Coumarin 334 and Rhodamine 590 dyes is around (506515)nm, and, (559-570), respectively. Initially it has been observed that the absorption and fluorescence intensity at maximum wavelength was increase with increasing concentration for two types of dye laser (Coumarin 334 and Rhodamine 590). This is because of low concentration--n adopted the dye molecules became more relaxed and there are a large intermolecular distance. in addition to the wavelength displacement (red shifting) in peaks position which is attributed to the self -absorption which modifies the observed fluorescence spectrum by reducing the intensity of the short wavelength region of spectrum (due to overlap with the absorption spectrum) and enhancing the intensity of the long wavelength region. 

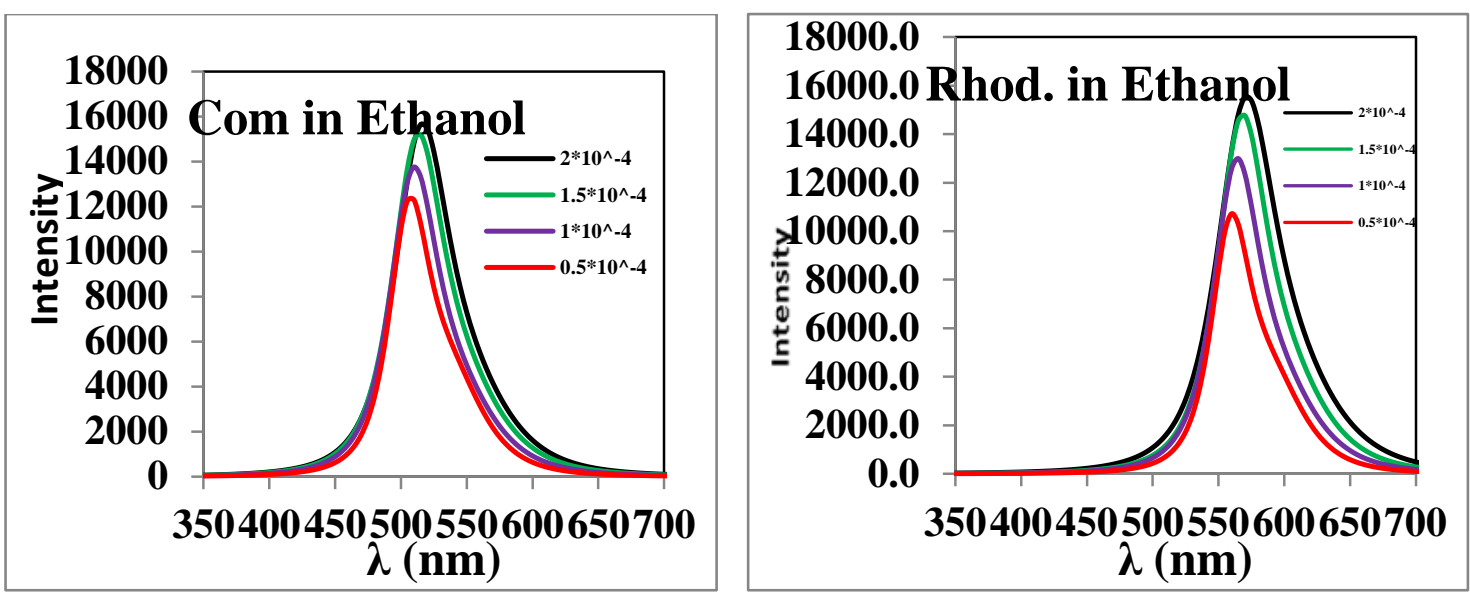

Fig. 5: The fluorescence spectrum of (a) Coumarin 334 (b) Rhodamin 590 dye solution.

To confirm the energy transfer process, the excitation spectrum of coumarin 334 dye should be plotted together with the emission spectrum of Rhodamin 590. The transfer process depend on the area of overlap since the area under the emission spectrum of acceptor Rhodamin 590 overlaps with the excitation spectrum of Coumarin so the transfer from coumarin 334 to Rhodamin 590 is happened Fig. 6 show this relation.

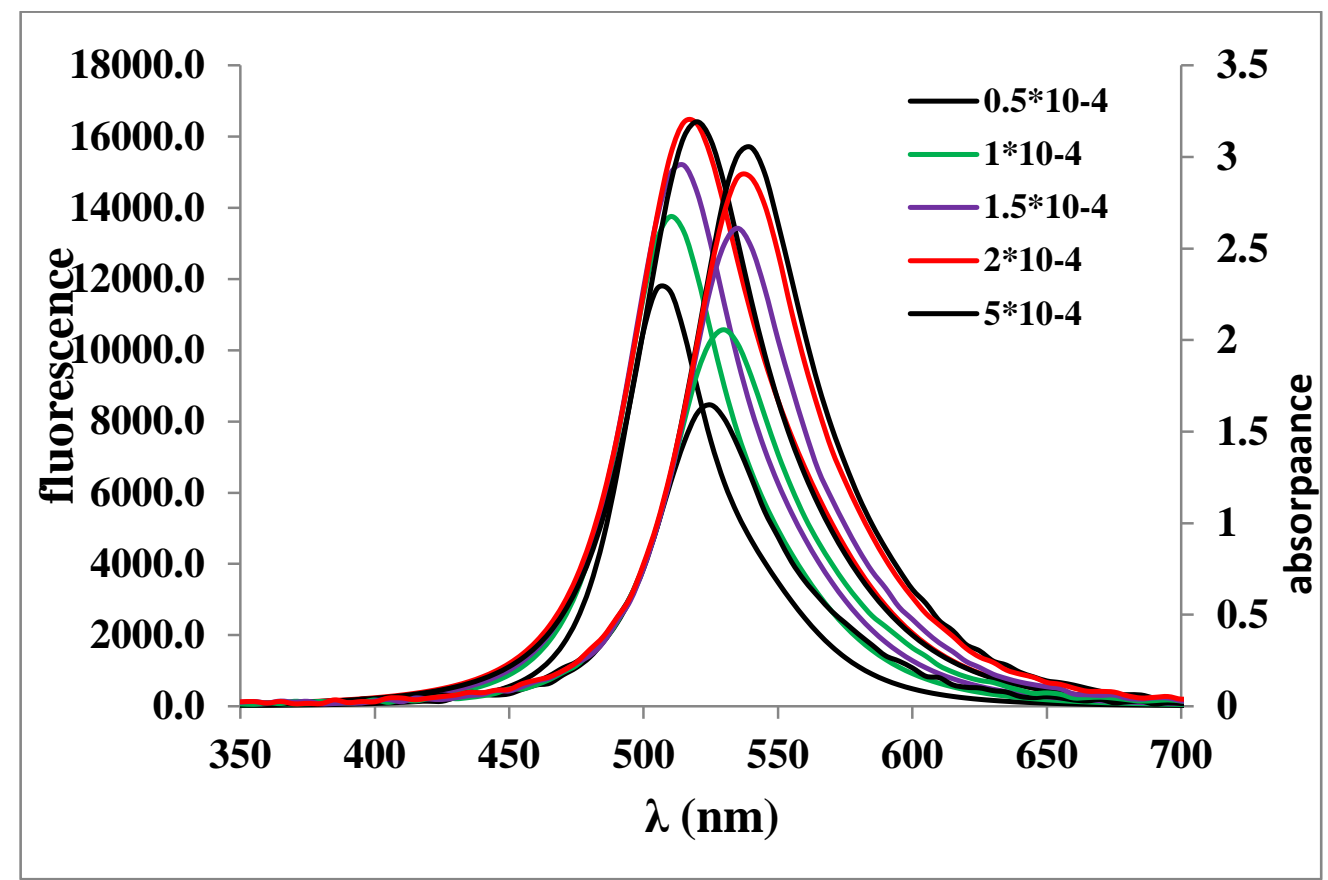

Fig. 6: The overlap between fluorescence spectra of coumarin 334 dye and absorption spectra of Rhodamine 590 dye solution at different molar concentration.

The spectral overlapping between the fluorescence band and the absorption band, is in the wavelength range of $455-605 \mathrm{~nm}$ it is appropriate for achieving the energy transfer process from Coumarin 334 as donor molecules to Rhodamin 590 as acceptor molecules.
The room temperature fluorescence spectra of dye mixture (10, 10 and 10)M of Coumarin 334 and at different concentration of Rhodamine590 doped as a solution are illustrated in Fig. 7 it can be observed from these figures that the fluorescence intensity of acceptor dyes Rhodamin 590 increases with the 
increase of its concentration at fixed donor concentration. This may be due to the reduction of intermolecular distance between the donor and acceptor molecules. One can see that the appearance of a small red shift of the peak emission wavelength of donor compared with acceptor as the acceptor dye concentration increases. This result gives an indication that the acceptor molecules Rhodamine 590 in dye mixture can be excited by two ways. The first one is by direct pumping because of the acceptor fluorescence enhancement at the acceptor concentration range in the present study. Secondly, by er-absorption of the energy from donor molecules Coumarin 334 in the energy transfer process, where the red shift of donor fluorescence spectral curves gives an indication that the excitation energy is transferred from donor to acceptor at the cost of emission from the fromer moleculer system.

The energy transfer rate constant $\left(\mathrm{K}_{\mathrm{ET}}\right)$ is estimated from stern-volmer relation and plots[13]

$$
\mathrm{I}_{\mathrm{D}} / \mathrm{I}_{\mathrm{DA}}=1+\mathrm{K}_{\mathrm{ET}} \mathrm{T}_{\mathrm{D}}[\mathrm{A}]
$$

here $I_{D}$ and $I_{D A}$ are the fluorescence intensities in the absence and presence of acceptor, respectively; [A] is the acceptor concentration $T_{D}$ is the fluorescence lifetime of donor in the absence of acceptor Fig. 8 show Stern -volmer plots of Coumarin 334 Rhodamin 590 dye mixture, respectively. This plot represents the relation of $\left(\mathrm{I}_{\mathrm{D}} / \mathrm{I}_{\mathrm{DA}}\right)$ as a function of acceptor concentration.

The fluorescence intensities ratio increase linearly with the acceptor concentration and thus Stern -Volmer relation of energy transfer has been implemented. thi value of $K_{\mathrm{ET}}$ con be calculated from the slop of straight line and found to be in the range of $10^{10}$ to $10^{9} \quad \mathrm{l} / \mathrm{mol} \mathrm{s}$. Thus the dominant mechanism of energy transfer process, for bimolecular system in silica matrices is non-radiatioin resonance energy transfer [14]. The fundamental quantity in Foresters theory of resonance energy transfer is the critical transfer distance $\left(\mathrm{R}_{0}\right.$ forster radius ).the values of $R_{0}$ depend on the half quenching concentration $[\mathrm{A}]_{1 / 2}$ which is the concentration of acceptor required to decrease the fluorescence of the donor by one half and is given as [15]:

$\mathrm{K}_{\mathrm{ET}}=1 / \mathrm{T}_{\mathrm{D}}[\mathrm{A}]_{1 / 2}$ 

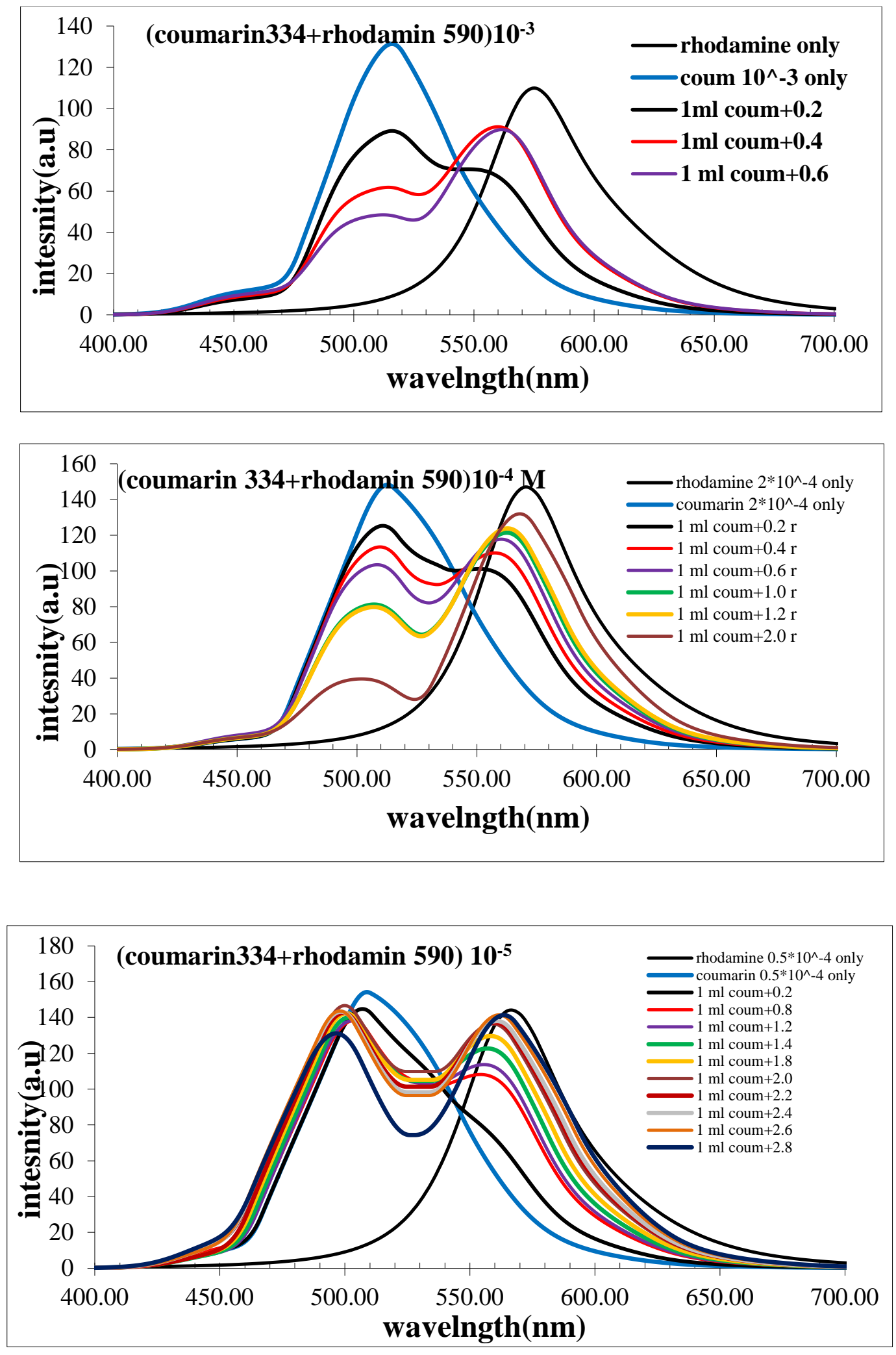

Fig. 7: Energy transfer process between Coumarin 334 and Rhodamin 590 at different concentration. 


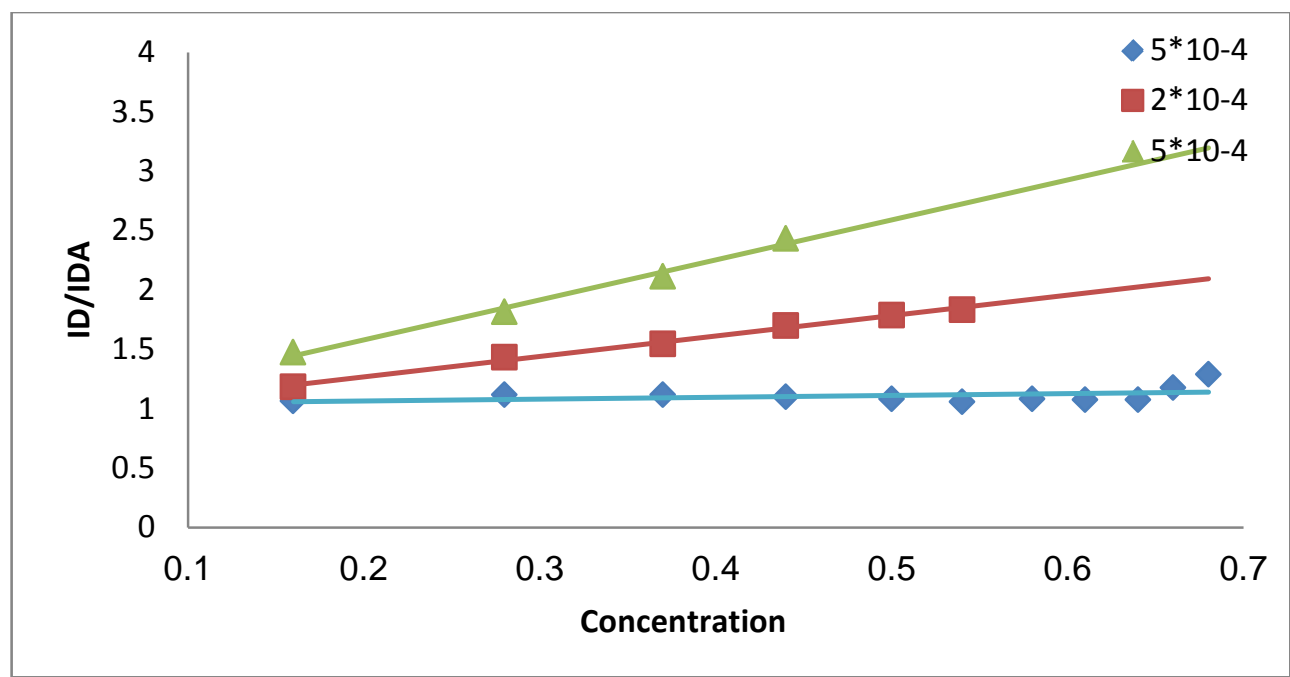

Fig. 8: Stern-Volmer plot for Coumarin 334 doped with Rhodamin 590 dye mixture in ethanol as a function of acceptor concentration.

Thus $\mathrm{R}_{0}$ (critical transfer distance) can be experimentally determined from the Stern-Volmewr plots using the relation [15]:

$$
\mathrm{R}_{\circ}=\frac{7.35}{\sqrt[3]{[\mathrm{A}]_{1 / 2}}}
$$

From Fig. 9 the value of $R_{0}$ is found to be in the range of $150 \mathrm{~A}^{\circ} \mathrm{R} 6 \mathrm{~A}$. since the range value of $\mathrm{R}$ more than $150 \mathrm{~A}$ represent the lower distance required for a radiation energy transfer and less than 6 A for collisional energy transfer [16] Therefore, the range value of $\mathrm{R}$ in the present study give another indication that the non- radiative resonance energy transfer is the dominant mechanism for bi- molecules in solution.

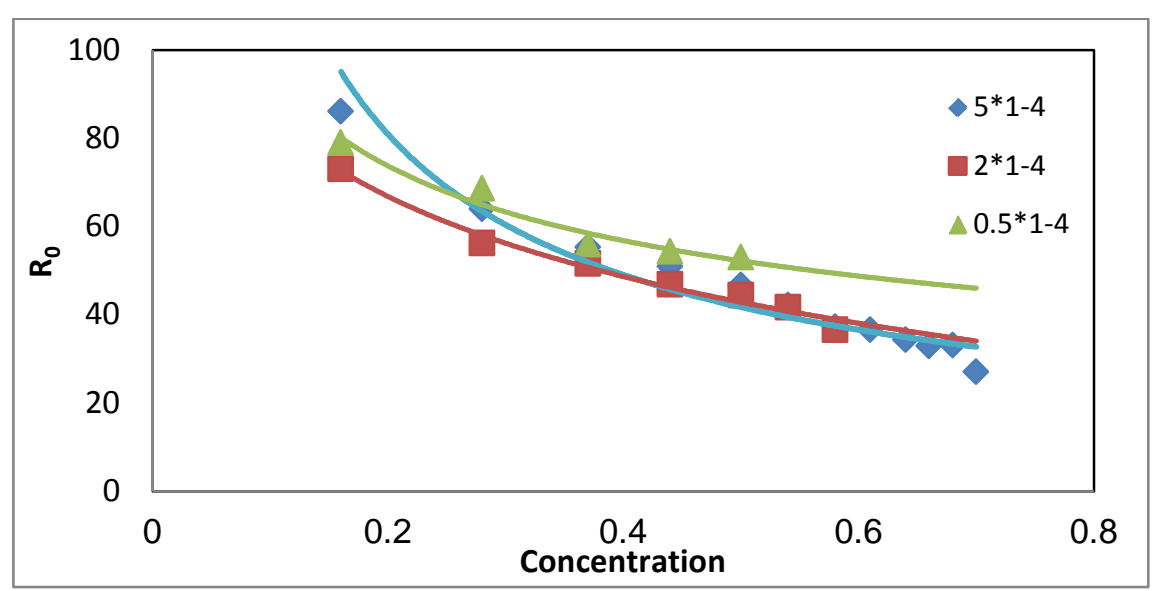

Fig. 9: $R_{o}$ as a function of acceptor concentration for different donor concentration.

\section{Conclusions}

The fluorescence spectra and energy transfer parameters for bi-molecules in solution indicate that non radiation resonance energy transfer is the dominant mechanism in such matrices. The dyes mixture encapsulated in solution leads to increase the acceptor emission intensity i.e. decreased bandwidth at FWHM. Thus, the aggregation effect of acceptor molecules and subsequently concentration quenching can be reduced compared with the case of single dye. 


\section{References}

[1] K. Uhlbrandt W \& DN. Wang, Nature 350, (1991) 130-134.

[2] R. Vav Grondelle, J.P. Dekker, T. Gillbro, V. Sundstr om, Biochim. Biophys. Acta Bioenerg. 1187 (1994) 1-65.

[3] R. Hildner D. Brinks J. B. Nieder, R.J Cogdell, N. F. Van Hulst, Science 340 (2013) 1448-1451

[4] D.J. Farrell and N.J. Ekins Daukes, Nat Photon, 3 (2009) 373-374.

[5] K. Shankar, X. Feng, C.A. Grimes, ACS Nano 3 (2009) 788-794.

[6] M.A. Baldo, M.E. Thompson, S.R. Forrest, Nature 403 (2000) 750-753

[7] I.L. Medintz, A.R. Clapp, H. Mattoussi, E. R. Goldman, B. Fisher, J.M. Mauro, Nat. Meter. 2, (2003) 630638.

[8] J. R. Heldt, JHeldt, M. Obarowska, B. Mielewska, J. Kamin skil, Journal of fluorescence, 11, 4 (2001) 335-342.

[9] I. B. Berlman, "energy transfer parameters of Aromatic compounds",
Academic London and New York, (1973).

[10] J. R. Lakowicz. "principles of fuorescence spectroscopy", Kluwer Academic/plenum publishers, New York, (1999).

[11] T. Forester, Ann.Physics, 2 (1984) 55-75 [translated by R.S.Knox].

[12] R. Rudolf, M. Mongillo, R. Rizzuto,T. Pizzan, Nat. Rev. Mol. Cell Biol. , 4, 7 (2003) 579-586.

[13] S. Singh, V. R. Kanetkar, G. Sridhar. V. Muthuswamy, K.R.aja, Journal of Lumin. 101 (2003) 285-291. [14] Mitsugu Terada and Yujiro Ohba, Jpn. J. Appl. Phys. 22, part 1, 9 (1983) 1392-1396.

[15] R.Ghazy, S.A.Zim, M.Shaheen, F.ElMekawey, Opt.Laser Technol.36, (2003) 463-469.

[16] B.B. Raju, Laser Chem.16 (1994) 109-120. 